\title{
MicroRNA-377 Inhibits Atherosclerosis by Regulating Triglyceride Metabolism Through the DNA Methyltransferase 1 in Apolipoprotein E-Knockout Mice
}

\author{
Ling-Yan Chen, MD; Xiao-Dan Xia, PhD; Zhen-Wang Zhao, MD; Duo Gong, PhD; \\ Xiao-Feng Ma, PhD; Xiao-Hua Yu, PhD; Qiang Zhang, MD; Si-Qi Wang, MD; Xiao-Yan Dai, PhD; \\ Xi-Long Zheng, PhD; Da-Wei Zhang, PhD; Wei-Dong Yin, PhD; Chao-Ke Tang, PhD
}

\begin{abstract}
Background: Lipoprotein lipase (LPL) plays an important role in triglyceride metabolism. It is translocated across endothelial cells to reach the luminal surface of capillaries by glycosylphosphatidylinositol-anchored high-density lipoprotein binding protein 1 (GPIHBP1), where it hydrolyzes triglycerides in lipoproteins. MicroRNA 377 (miR-377) is highly associated with lipid levels. However, how miR-377 regulates triglyceride metabolism and whether it is involved in the development of atherosclerosis remain largely unexplored.

Methods and Results: The clinical examination displayed that miR-377 expression was markedly lower in plasma from patients with hypertriglyceridemia compared with non-hypertriglyceridemic subjects. Bioinformatics analyses and a luciferase reporter assay showed that DNA methyltransferase 1 (DNMT1) was a target gene of miR-377. Moreover, miR-377 increased LPL binding to GPIHBP1 by directly targeting DNMT1 in human umbilical vein endothelial cells (HUVECs) and apolipoprotein E (ApoE)-knockout (KO) mice aorta endothelial cells (MAECs). In vivo, hematoxylin-eosin (H\&E), Oil Red O and Masson's trichrome staining showed that ApoE-KO mice treated with miR-377 developed less atherosclerotic plaques, accompanied by reduced plasma triglyceride levels.
\end{abstract}

Conclusions: It is concluded that miR-377 upregulates GPIHBP1 expression, increases the LPL binding to GPIHBP1, and reduces plasma triglyceride levels, likely through targeting DNMT1, inhibiting atherosclerosis in ApoE-KO mice.

Key Words: Atherosclerosis; DNA methyltransferase 1 (DNMT1); Glycosylphosphatidylinositol-anchored high-density lipoproteinbinding protein 1 (GPIHBP1); Lipoprotein lipase (LPL); MicroRNA-377

\begin{abstract}
A therosclerosis is the common pathological basis of cardiovascular and cerebrovascular diseases. Lipid metabolism disorder, such as the aberrant elevation of triglyceride levels, is an independent risk factor for atherosclerosis. ${ }^{\mathbf{1} 2}$ Increasing evidence suggests that microRNAs (miRNAs) play a critical role in lipid metabolism and atherosclerosis. ${ }^{3,4}$ Previously, our colleagues showed that miR-186, miR-134, and miR-467b induce lipid accumulation and activate an inflammatory response in macrophages through regulating lipoprotein lipase (LPL) ${ }^{5-7}$ MicroRNA-377 (miR-377), a polyphonic miRNA, is known to be involved in oxidative stress, inflammation, angiogenesis in ischemic hearts, and the cardiac regenerative
\end{abstract}

ability. ${ }^{\mathbf{8 - 1 0}}$ In addition, miR-377 levels are closely associated with lipid levels in vivo, indicating its role in regulating lipid metabolism. ${ }^{11,12}$ However, the molecular mechanisms for miR-377-modulated triglyceride metabolism in the development of atherosclerosis are poorly studied.

DNA methylation, one of the earliest ways of epigenetic modifications, exists in all higher organisms. ${ }^{13,14}$ The cytosine rings of $\mathrm{CpG}$ sequence are subjected to methylation modification in response to DNA methyltransferases (DNMTs), leading to silencing of gene expression. ${ }^{13,14}$ DNMT1, one of the most important DNA methyltransferases, is responsible for maintaining the methylated state of DNA after replication, which allows the methylation

Received April 17, 2018; revised manuscript received July 22, 2018; accepted August 3, 2018; released online September 20,2018 Time for primary review: 29 days

Institute of Cardiovascular Research, Key Laboratory for Atherosclerology of Hunan Province, Medical Research Center, Hunan Province Cooperative Innovation Center for Molecular Target New Drug Study (L.-Y.C., X.-D.X., Z.-W.Z., D.G., X.-H.Y., Q.Z, S.-Q.W., W.-D.Y, C.-K.T.), Department of Internal Medicine-Cardiovascular, Nanhua Hospital (X.-F.M.), University of South China, Hengyang, Hunan; Key Laboratory of Molecular Target \& Clinical Pharmacology, School of Pharmaceutical Sciences \& the Fifth Affiliated Hospital, Guangzhou, Medical University, Guangzhou (X.-Y.D.), China; Department of Biochemistry and Molecular Biology, Libin Cardiovascular Institute of Alberta, Cumming School of Medicine, University of Calgary, Health Sciences Center, Calgary, Alberta (X.-L.Z.); and Department of Pediatrics and Group on the Molecular and Cell Biology of Lipids, University of Alberta, Alberta (D.-W.Z.), Canada

The first three authors contributed equally to this work (L.-Y.C., X.-D.X., Z.-W.Z.).

Mailing address: Wei-Dong Yin, PhD or Chao-Ke Tang, PhD, Institute of Cardiovascular Research, University of South China, 28 W Changsheng Road, Hengyang, Hunan 421001, China. E-mail: wdy20042004@126.com; tangchaoke@qq.com

ISSN-1346-9843 All rights are reserved to the Japanese Circulation Society. For permissions, please e-mail: cj@j-circ.or.jp 
pattern to be passed on to the progeny cells. ${ }^{14,15}$ Overexpression of DNMT1 in macrophages induces pro-inflammatory cytokines production and atherosclerosis development ${ }^{16}$ Moreover, DNMT1 is associated with elevated triglyceride levels. ${ }^{17,18}$ However, the substrate of DNMT1 involved in triglyceride metabolism has not yet been fully elucidated.

LPL plays an important role in triglyceride metabolism. ${ }^{19,20}$ After secretion by parenchymal cells (e.g., cardiomyocytes and adipocytes), LPL is translocated across endothelial cells to reach the luminal surface of capillaries by glycosylphosphatidylinositol-anchored high-density lipoprotein (HDL) binding protein 1 (GPIHBP1), where it hydrolyzes triglycerides (TG) in lipoproteins. ${ }^{19,21-23}$ Some studies have shown that GPIHBP1 stabilizes LPL and prevented its inhibition of angiopoietin-like 3 (ANGPTL 3) and angiopoietin-like 4 (ANGPTL 4), but GPIHBP1 has no effect on the LPL activity. ${ }^{24}$ Recently, many noteworthy clinical studies reported that patients with mutations in GPIHBP1 are suffered from severe hypertriglyceridemia..$^{25,26}$ Furthermore, it has been reported that GPIHBP1 autoantibodies block the ability of GPIHBP1 to bind and transport LPL, which interferes with the LPL-mediated procession of triglyceride-rich lipoproteins and consequently causes severe hypertriglyceridemia. ${ }^{27}$ Global deletion of GPIHBP1 in mice leads to elevated plasma levels of triglyceride. ${ }^{22}$ Therefore, GPIHBP1 is an important regulator in reducing plasma triglyceride levels. However, the regulatory mechanism of GPIHBP1 expression, especially epigenetic regulation of GPIHBP1, is still largely unknown.

Here, we reported that patients with hypertriglyceridemia showed reduced plasma levels of miR-377 mRNA. Overexpression of $\mathrm{miR}-377$ reduces triglyceride levels and atherosclerotic plaques in apolipoprotein E (ApoE)knockout (KO) mice. Mechanistically, miR-377 downregulated DNMT1, which increased the binding of LPL to GPIHBP1 and consequently reduces plasma triglyceride levels. Taken together, the results suggest that miR-377 may act as a novel pharmacologic target for atherosclerosis therapy.

\section{Methods}

\section{Cell Culture}

Human umbilical vein endothelial cells (HUVECs) and HEK 293T cells were obtained from Cell Bank of the Chinese Academy of Sciences (Shanghai, China). HUVECs were maintained at $37^{\circ} \mathrm{C}$ using an endothelial cell growth medium 2 (EGM-2) bullet kit supplemented with $2 \%$ fetal bovine serum (FBS), endothelial cell growth supplement, $50 \mu \mathrm{g} / \mathrm{mL}$ heparin, $100 \mathrm{IU} / \mathrm{mL}$ penicillin, $100 \mu \mathrm{g} / \mathrm{mL}$ streptomycin, $2.5 \mu \mathrm{g} / \mathrm{mL}$ amphotericin $\mathrm{B}$, and other supplements (Lonza, Switzerland). The endothelial cell growth supplement contains vascular endothelial growth factor (VEGF), human fibroblast growth factor-B (FGF-B), insulin-like growth factor 1 (IGF-1), and epidermal growth factor (EGF) (Lonza, Switzerland). The other supplements include hydrocortisone, ascorbic acid, gentamicin and insulin (Lonza, Switzerland). HEK 293T cells were maintained as adherent monolayers by serial passage in Dulbecco's modified Eagle's medium (DMEM) (Hyclone, USA) containing 10\% FBS. All HUVECs and HEK 293T cells were maintained in an incubator with a saturated humidity environment of $95 \%$ air $-5 \% \mathrm{CO}_{2}$ at $37^{\circ} \mathrm{C}$.

\section{Western Blot Analysis}

Cells were lysed in RIPA buffer (Sigma, St Louis, MO, USA) on ice for protein extraction, as described previously. ${ }^{19,28}$ The protein content was assayed by BCA protein assay reagent (Pierce, USA). A sample of $50 \mu \mathrm{g}$ protein was loaded to SDS-PAGE, and then transferred to the polyvinylidene difluoride (PVDF) (Millipore Corporation, USA) membranes. The membrane was blocked with 5\% non-fat dry milk (NFDM) in TBST (Tris $0.05 \%$ Tween-20) at $4{ }^{\circ} \mathrm{C}$ for $4 \mathrm{~h}$. Both GPIHBP1 and LPL antibodies were purchased from Abcam (Cambridge, UK), and $\beta$-actin antibody (AF0003) from Beyotime. The membrane was incubated with a 1:1,000 dilution of primary antibody overnight at $4^{\circ} \mathrm{C}$, washed with TBST and incubated with a 1:2,500 dilution of horseradish peroxidase (HRP)conjugated secondary antibody diluted in TBST containing $5 \% \mathrm{NFDM}$ at room temperature for $2 \mathrm{~h}$. Immunoreactive bands were visualized with Tanon-5500 Chemiluminescent Imaging System (Tanon, China) and BeyoECL Plus (Beyotime, China).

\section{Real-Time Quantitative PCR (qPCR) Analysis}

qPCR analysis was performed as described previously. ${ }^{28}$ Cells total RNA was extracted using TRIzol reagent (Invitrogen, Carlsbad, CA, USA) according to the manufacturer's introduction. Reverse transcription was conducted by a High-Capacity cDNA reverse transcription kit (Takara). qPCR was performed by SYBR Green detection chemistry with a Light Cycler Run 5.32 Real-Time PCR System (Roche, Swiss). The analyses of all qPCR Melt curve analyses were performed and showed to produce a single DNA duplex. Quantitative measurements were confirmed by the $\Delta \Delta \mathrm{Ct}$ method. In these experiments, gene expression data were normalized to GAPDH levels. The primers and their sequences are shown in Table S1.

\section{Bisulfite Sequencing of the GPIHBP1 Promoter}

DNA was extracted from cells using the Dneasy Kit (Gibco, USA), according to the manufacturer's instruction. Bisulfite modification was executed by using an EZ DNA Methylation-Direct ${ }^{\mathrm{TM}}$ Kit (Zymo Research, USA). After bisulfite conversion, the GPIHBP1 promoter was amplified with the following primers: forward, 5'-CGGTGCCAAC GGGCGGATGGTC-3', and reverse, 5'-GCCACGGTTG CCCGCCTACCAG-3'. PCR was performed with an EpiTaq HS enzyme (Takara, JP). After purified using a QIAquick Gel Extraction Kit (Qiagen Inc., CA), the PCR fragment was cloned into the TA vector (Invitrogen, USA). The sequences were determined with an ABI PRISM ${ }^{\circledR}$ 3700 Genetic Analyzer (Applied Biosystems, USA), and data were summarized by using the web-based software, QUMA (http://quma.cdb.riken.jp/). After, the chi-squared test was used to analyze the rate of each methylation variation.

\section{Binding of LPL to GPIHBP1 Assay}

After treated with PIPLC, cells were treated with miR-377 mimic or miR-377 inhibitor. Twenty-four hours later, the GPIHBP1-expressing HUVECs continue to grow on fibronectin-coated plates or coverslips were incubated with Flag-tagged human LPL for $2 \mathrm{~h}$ at $4^{\circ} \mathrm{C}$. After washing extensively in $1 \times \mathrm{PBS}$, cells were treated with heparin $(250 \mathrm{U} / \mathrm{mL})$ to release surface-bound LPL. The cell lysates were collected and the heparin-released LPL was examined for the binding of LPL to GPIHBP1 by Western blot. 


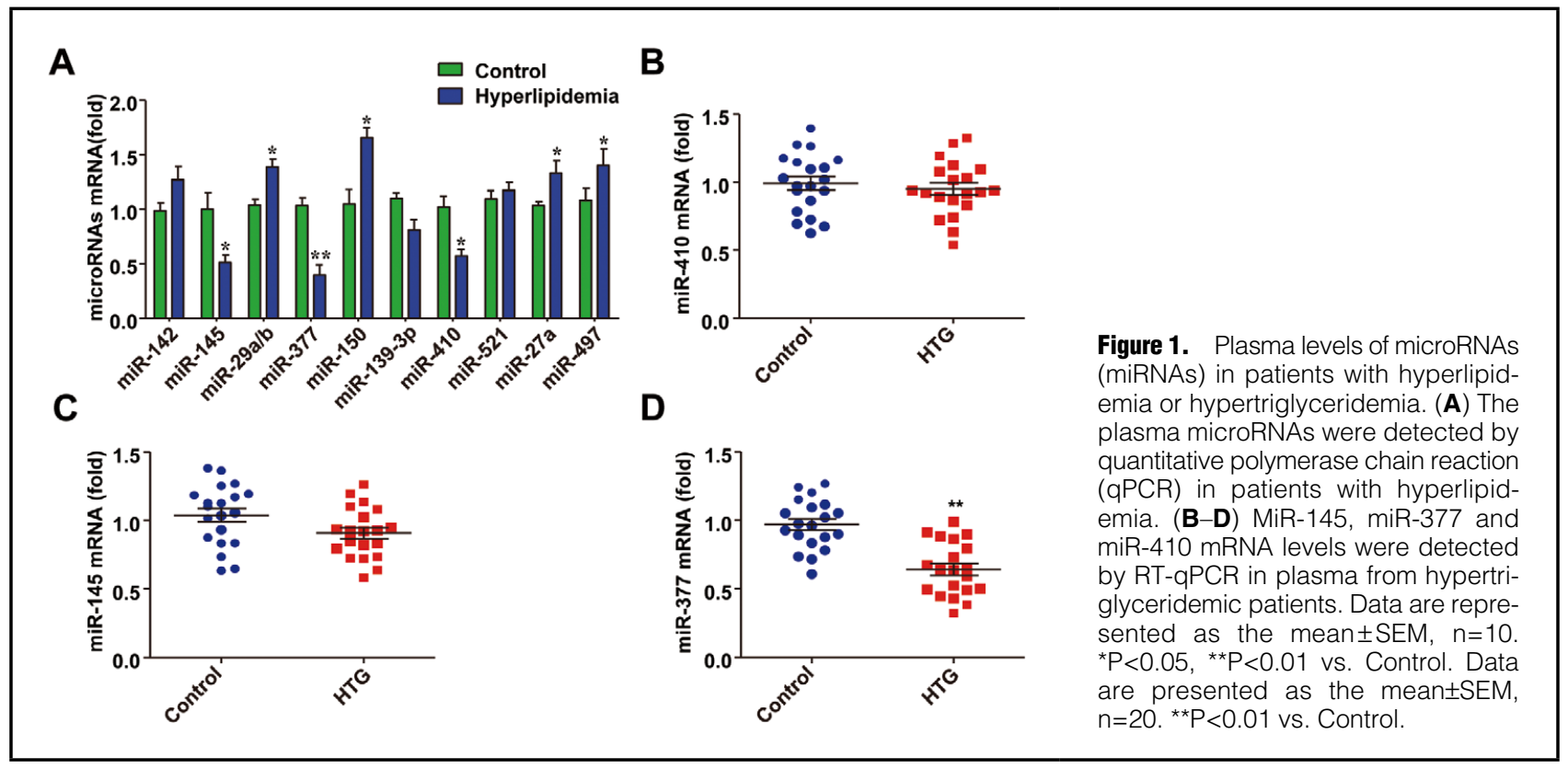

\section{Animal Studies}

Male 8-week-old ApoE-KO mice were purchased from Changzhou Laboratory Animal Center (Cavens Experimental Animal CO., Ltd, Jiangsu, China). Mice were randomly divided into 4 groups ( $=10$ mice/group): miR-377 scrambled miR-377 agomir negative control (AG-NC), miR-377 agomir (AG), miR-377 antagomir negative control (ANNC), and miR-377 antagomir (AN). The agomir (2'OME+ 5' chol modified) and antagomir (2'OME+5' chol modified) of miR-377 were purchased from RiboBio (Guangzhou, China) with sequences shown below: miR-377 agomir negative control: UCACAACCUCCUAGAAAGAGUAGA, miR-377 agomir: AGAGGUUGCCCUUGGUGAAUUC, miR-377 antagomir negative controls: UUUGUACUAC ACAAAAGUACUG, and miR-377 antagomir: AUCAC ACAAAGGCAACUUUUGU. Mice were fed a high-fat/ high-cholesterol diet (Trophic Animal Feed High-tech Co., Ltd, Jiangsu, China) and received a tail vein injection of miR-377 agomir, miR-377 antagomir or one of the corresponding controls at a dose of $80 \mathrm{mg} / \mathrm{kg}$ wt in $0.2 \mathrm{~mL}$ saline once every 2 weeks. All mice were maintained in a specific pathogen-free environment with free access to food and water. After 12 weeks on the high-fat/high-cholesterol diet, mice were fasted for $12-14 \mathrm{~h}$ and anesthetized with $1 \%$ Pelltobarbitalum Natricum (Yubo Biological, Shanghai, China). The aortic sinus sections were sectioned by using a rapidly frozen sections technique. The plaque area, lipid accumulation and collagen content in the aortic sinus were detected by HE, Oil red O, and Masson's trichrome staining respectively. All mice were housed in a controlled environment $\left(20 \pm 2^{\circ} \mathrm{C}, 12-\mathrm{h} / 12\right.$-h light/dark cycle $)$. All procedures were carried out in accordance with the Institutional Animal Ethics Committee and the University of South China Animal Care Guidelines for the Use of Experimental Animals.

\section{Statistical Analysis}

Quantitative values are expressed as the mean \pm SEM and represent data from at least 3 independent experiment. The difference between 2 groups was analyzed by using a Student's t-test. The difference by Newman-Keus multiple comparison tests and comparisons of different parameters between each group were made by 2-way ANOVA analysis followed by Bonferroni post-tests. A P value of less than 0.05 was considered statistically significant.

\section{Results}

Patients With Hypertriglyceridemia Show Reduced Plasma miR-377 Levels

Clinical characteristics of 550 patients (mean age, 69.1 years; 61\% male) are shown in Table S2. Patients with hyperlipidemia exhibited higher plasma levels of total cholesterol (TC) and triglyceride and fasting blood glucose levels compared to those without hyperlipidemia. Of note, serum triglyceride levels were significantly higher in patients with hyperlipidemia relative to those without hyperlipidemia (median, $2.51 \mathrm{mmol} / \mathrm{L}$ [interquartile range, $1.81-3.38 \mathrm{mmol} / \mathrm{L}$ ] vs. $1.17 \mathrm{mmol} / \mathrm{L}$ [interquartile range, 0.76-1.45]; $\mathrm{P}<0.001$ ) (Table S2).

To investigate the association of microRNAs with dyslipidemia, we determined plasma levels of 10 lipid metabolism-associated miRNAs in patients by using quantitative real-time PCR. As shown in Figure 1A, the expression of miR-145, miR-377, and miR-410 was markedly decreased in patients with dyslipidemia, including hypertriglyceridemia, hypercholesterolemia, hypo-HDL cholesterolemia and complex hyperlipidemia with combined phenotypes (Figure 1A). We further determined the levels of miR-145, miR-377, and miR-410 in plasma of patients with hypertriglyceridemia to assess their association. Interestingly, only miR-377 expression was significantly lower in patients with hypertriglyceridemia (Figure 1B-D).

\section{miR-377 Binds to DNMT1's 3'-Untranslated Region (3'-UTR) and Inhibits DNMT1 Expression and Activity}

Analysis using microRNA.org-Targets and Expression revealed a predicted binding site of miR-377 on the 3'-UTR of DNMT1, which is highly conserved among different species (Figure S1A). Several target gene prediction algorithms also suggest a highly homologous sequence of 

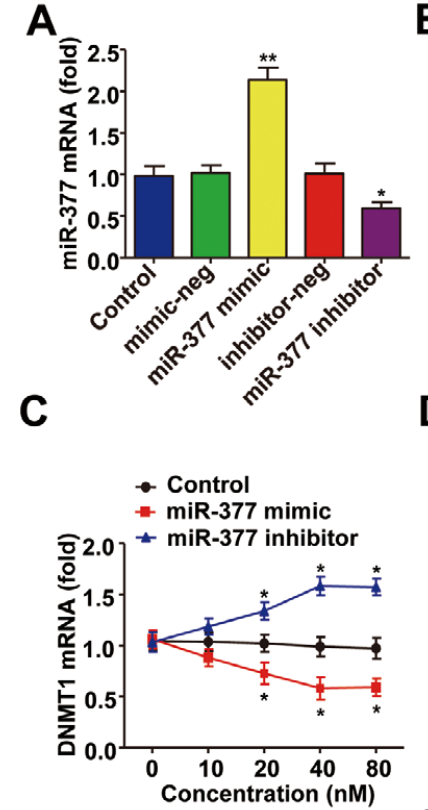

$\mathbf{F}$

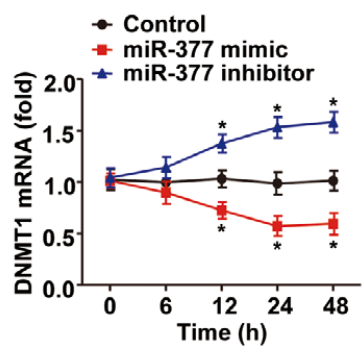

B
D

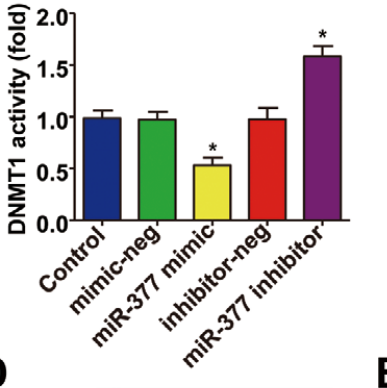

E

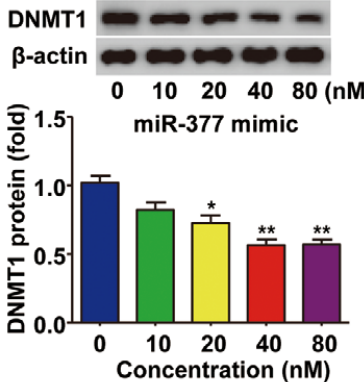

G

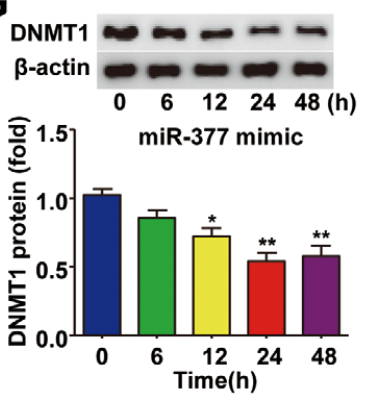

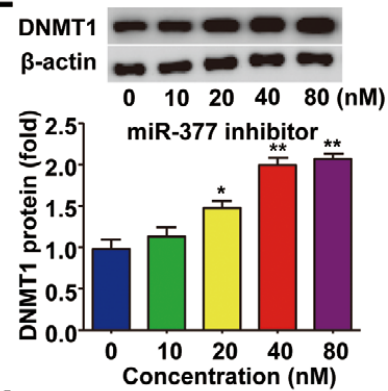

H
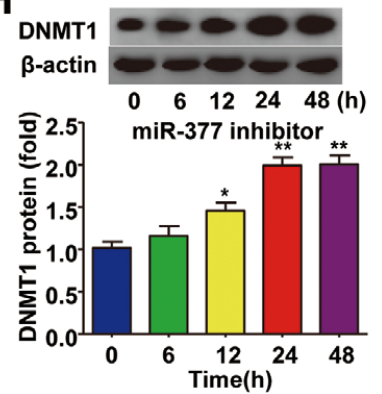

Figure 2. Effects of microRNA-377 (miR-377) on DNA methyltransferase 1 (DNMT1) activity and expression in human umbilical vein endothelial cells (HUVECs). (A) HUVECs were treated with miR-377 mimic or miR-377 inhibitor and then miR-377 expression was detected by qPCR. (B) DNMT1 activity was quantified in HUVECs treated with miR-377 mimic or miR-377 inhibitor for $24 \mathrm{~h}$. (C-E) DNMT1 mRNA and protein expression were detected when cells were treated with miR-377 mimic or miR-377 inhibitor for the indicated concentrations. (F-H) DNMT1 mRNA and protein expression when cells were treated with miR-377 mimic or miR-377 inhibitor for the indicated time periods. Data are presented as the mean $\pm \mathrm{SEM}, \mathrm{n}=3 .{ }^{*} \mathrm{P}<0.05,{ }^{* *} \mathrm{P}<0.01 \mathrm{vs}$. Control and ${ }^{*} \mathrm{P}<0.05$, ${ }^{* *} \mathrm{P}<0.01$ vs. $0 \mathrm{nmol} / \mathrm{L}$ or $0 \mathrm{~h}$ respectively.

the miR-377 binding site in the 3'-UTR of human DNMT1 mRNA (Targetscan, miRDB, and microRNA.org) that is also highly conserved among different species (Figure S1B). Further, the free energy scores (miRnaViewer and RNAhybrid) for the hybridization between miR-377 and DNMT1 are low in humans (Figure S1C). Together, these predictions suggest that miR-377 may be a potentially important regulator of DNMT1. To investigate this possibility, we constructed a luciferase reporter vector containing wild-type DNMT1 3'-UTR with the miR-377 homologous sequence or a mutant DNMT1 3'-UTR, in which the miR-377 homologous sequence was eliminated (Figure S1D). When compared with the control in HEK 293T cells, miR-377 mimic had no effect on the luciferase activity of GPIHBP1 and LPL (Figure S1F,G) but significantly decreased the relative luciferase activity of the wildtype DNMT1 3'-UTR reporter, while had no effect on the mutant DNMT1 3'-UTR (Figure S1E).

Next, we examined the effects of miR-377 on the activity and expression of DNMT1 in cells. As shown in Figure 2A, overexpression of miR-377 mimic markedly increased its mRNA levels and significantly reduced the activity of DNMT1. An opposite phenotype was observed when the cells were treated miR-377 inhibitor; miR-377 levels were reduced but DNMT1 activity was increased (Figure 2B). Furthermore, miR-377 mimic reduced, while miR-377 inhibitor increased, the mRNA and protein levels of DNMT1 mRNA and protein in endothelial cells in a concentration-dependent manner (Figure 2C-E). Consistently, the effects of miR-377 mimic and inhibitor on DNMT1 expression were time-dependent (Figure 2F-H). Collectively, these findings indicate that miR-377 repressed DNMT1 activity and expression potentially by targeting 3'-UTR of DNMT1 in HUVECs.

\section{DNMT1 Catalyzes GPIHBP1 Promoter Methylation and Attenuates Its Expression}

$\mathrm{CpG}$ islands are mainly located in the promoter and the 


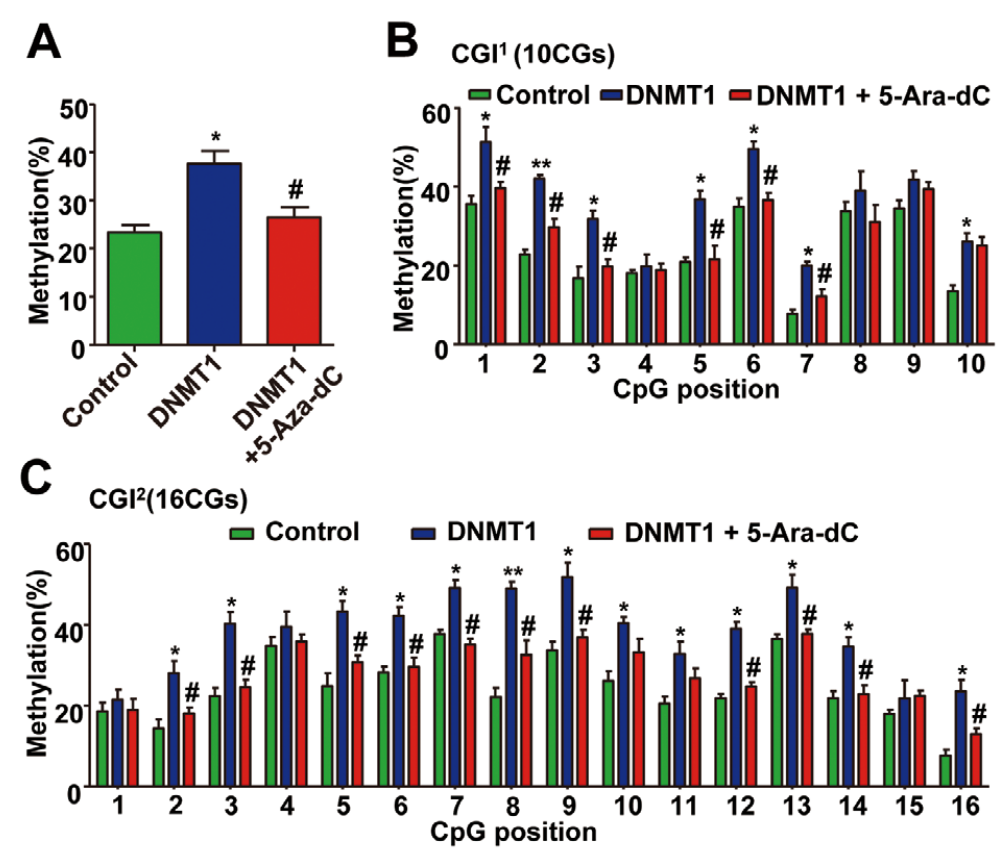

D

E
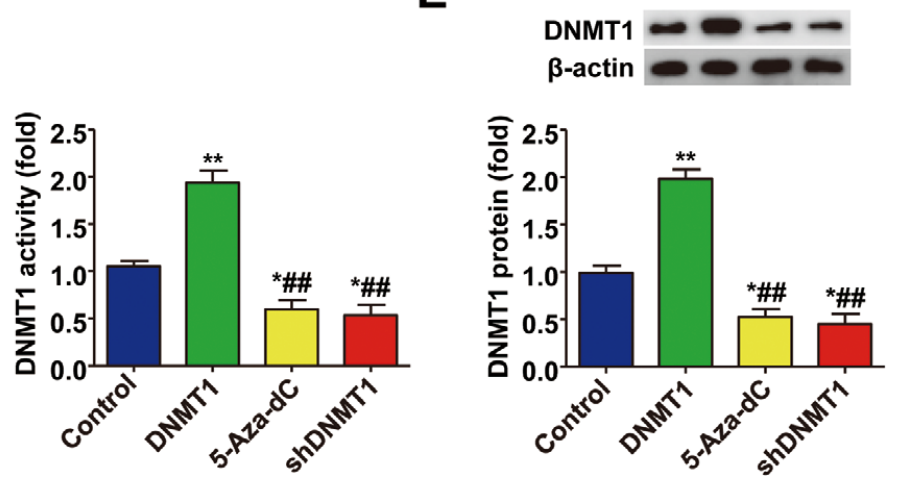

$\mathbf{F}$

G
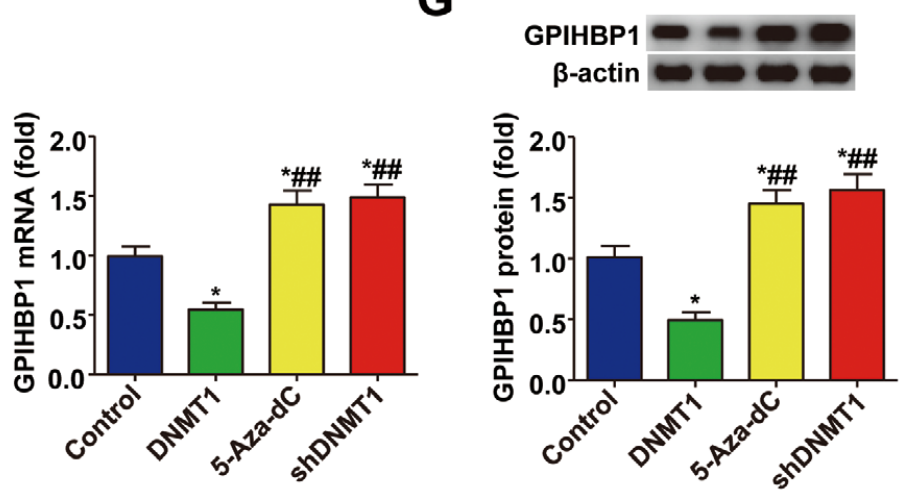

Figure 3. DNA methyltransferase 1 (DNMT1) mediates glycosylphosphatidylinositol-anchored high-density lipoprotein-binding protein 1 (GPIHBP1) promoter methylation and negatively regulates GPIHBP1 expression. (A) The overall methylation levels of GPIHBP1. The human umbilical vein endothelial cells (HUVECs) were treated by DNMT1 alone or co-treated with DNMT1 and 5-AZA-2'-deoxycytidine (5-AzaDc) $(10 \mu \mathrm{mol} / \mathrm{L})$ for $6 \mathrm{~h}$. (B) The methylation levels within the first of $\mathrm{CpG}$ islands $\left(\mathrm{CGI}^{1}\right)$ in the GPIHBP1 promoter region. (C) The methylation levels within the second of $\mathrm{CpG}$ islands (CGI $\left.{ }^{2}\right)$ in the GPIHBP1 promoter region. (D) 5-Aza-dC $(10 \mu \mathrm{mol} / \mathrm{L})$ or short hairpin RNADNMT1 (shDNMT1) downregulated DNMT1 activity. (E) 5-Aza-dC or shDNMT1 repressed DNMT1 protein expression. DNMT1 activity and protein were quantified and normalized in HUVECs transfected with DNMT1, 5-Aza-Dc, or shDNMT1 for $6 \mathrm{~h}$ as compared with the control. $(\mathbf{F}, \mathbf{G})$ Either 5-Aza-dC or shDNMT1 blocked DNMT1induced suppression of GPIHBP1 mRNA and protein expression. Data are presented as the mean \pm SEM, $n=3$. * $P<0.05,{ }^{*} P<0.01$ vs. Control and ${ }^{P}<<0.05, \# P<0.01$ vs. DNMT1.

first exon region of genes. We employed the UCSC Human Genome Browser and NCBI gene bank (http://www.ncbi. nlm.nih.gov/pubmed/) to define the genomic features of GPIHBP1 promoter sequence. We found that the GPIHBP1 promoter contains 2 well-defined genic CGIs clustered with a high density of $\mathrm{CpG}$ locus, as analyzed with online tools (https://www.ebi.ac.uk/services) (Figure S2A,B). The GPIHBP1 CGI ${ }^{1}$ and $\mathrm{CGI}^{2}$ are extended across $328 \mathrm{bp}$ and 318 bp containing a CG content of $62.8 \%$ and $59.7 \%$, with an observed-to-expected $\mathrm{CpG}$ ratio of 0.99 and 0.78 respectively (Table S3). Detailed sequence analysis indicates the presence of a central region that covers the midsection 
A
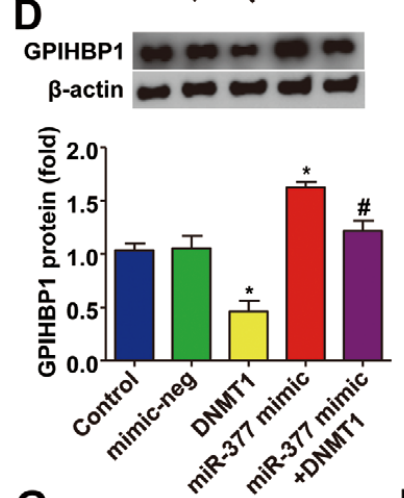

G

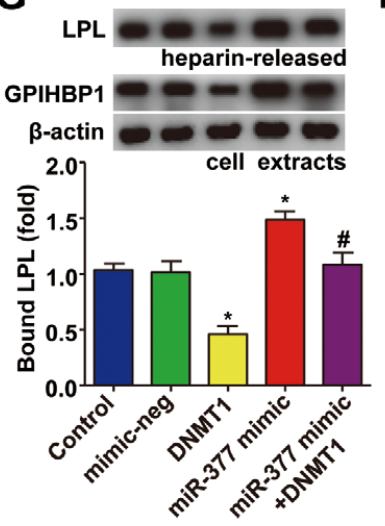

B

E
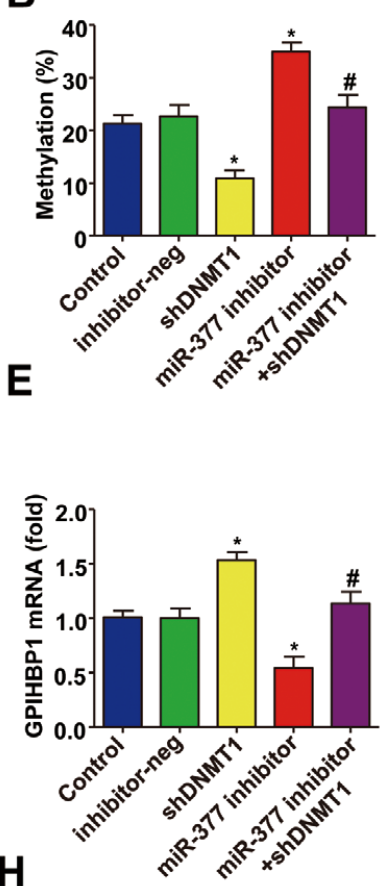

H

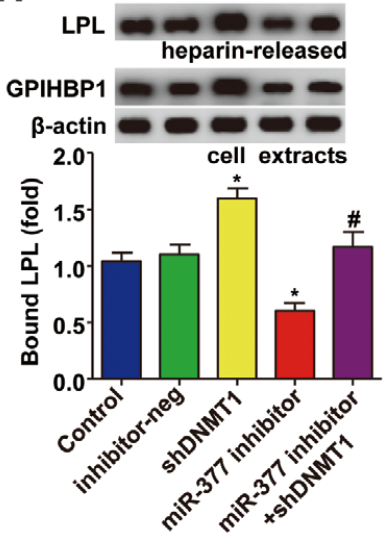

C

F
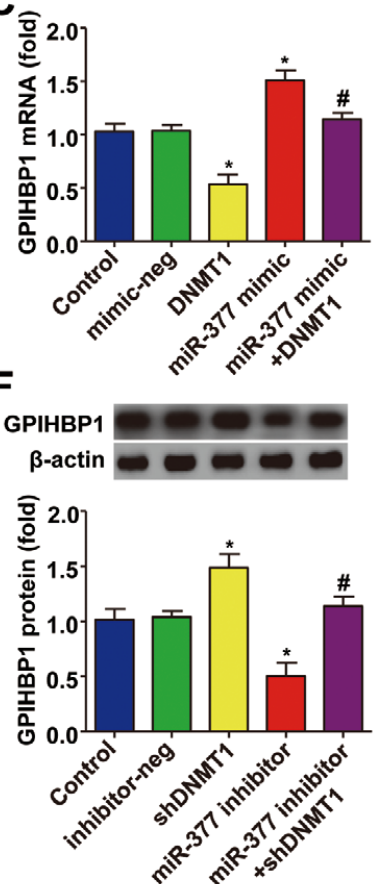

Figure 4. MicroRNA-377 (miR-377) mediates the binding of lipoprotein lipase (LPL) to glycosylphosphatidylinositol-anchored high-density lipoprotein binding protein 1 (GPIHBP1) by inhibiting DNA methyltransferase 1 (DNMT1). (A,B) GPIHBP1 promoter methylation levels were analyzed when cells were transfected with miR-377 mimic, DNMT1, miR-377 mimic+DNMT1 and miR-377 inhibitor, shDNMT1, and miR-377 inhibitor+shDNMT1 through bisulfite sequencing. (C,D) GPIHBP1 mRNA and protein expression were detected by quantitative polymerase chain reaction (GPCR) or western blotting, and treated with miR-377 mimic together with mimic-neg or DNMT1 as indicated respectively. (E,F) GPIHBP1 mRNA and protein expression were, respectively, examined by qPCR or western blotting and treated with miR-377 inhibitor together with inhibitor-neg or DNMT1 as indicated. (G,H) miR-377 increases the binding of LPL to GPIHBP1 by inhibiting DNMT1. The heparin-released LPL was examined for the binding of LPL to GPIHBP1 by western blotting (top row). Data are presented as the mean $\pm S E M, n=3 .{ }^{*} P<0.05$ vs. Control and $\# P<0.05$ vs. DNMT1 or shDNMT1 respectively.

with a high $\mathrm{CpG}$ load, indicating that GPIHBP1 promoter might be a target of DNA methylation.

To assess the role of DNMT1 in regulating the methylation of GPIHBP1 in HUVECs, we detected the methylation levels of different CG locus in the GPIHBP1 promoter by using bisulfite sequencing. We found that the methylation levels of the GPIHBP1 promoter were significantly increased with DNMT1 treatment, which was blocked by inhibition of DNMT1 with 5-Aza-dC (Figure 3A). Detailed analyses revealed that DNMT1 significantly increased
DNA methylation levels within the GPIHBP1 promoter at sites $1,2,3,5,6,7$ and 10 of $\mathrm{CGI}^{1}$, and at sites 2, 3, 5, $6,7,8,9,11,12,13$ and 15 of $\mathrm{CGI}^{2}$ (Figure 3B,C). The increase of DNA methylation levels in these sites, except for site 10 in $\mathrm{CGI}^{1}$, could be blocked by inhibition of DNMT1, indicating the important role of DNMT1 in DNA methylation of GPIHBP1 (Figure 3B,C). Although there was no difference at some sites, the methylation level of the GPIHBP1 promoter was totally increased in response to DNMT1 treatment (Figure 3A). 
A

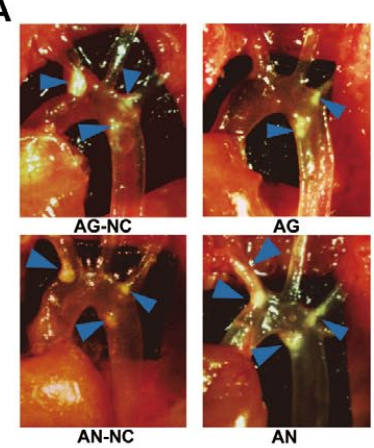

B

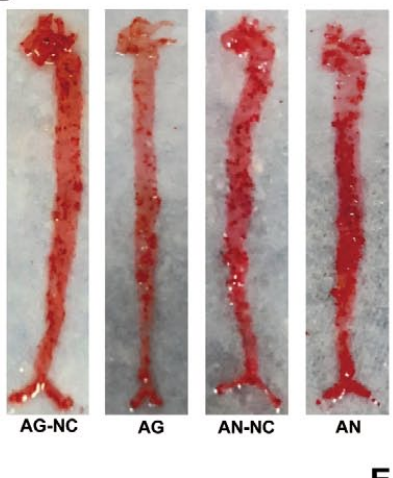

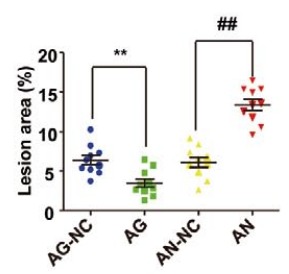

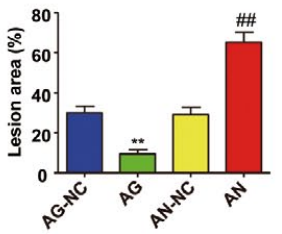

E

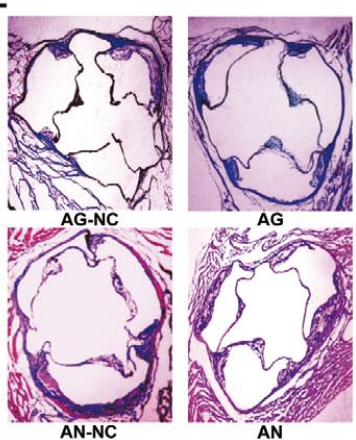

C
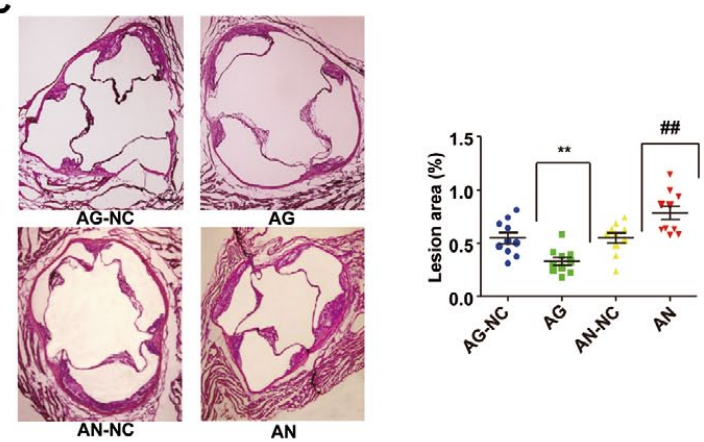

D
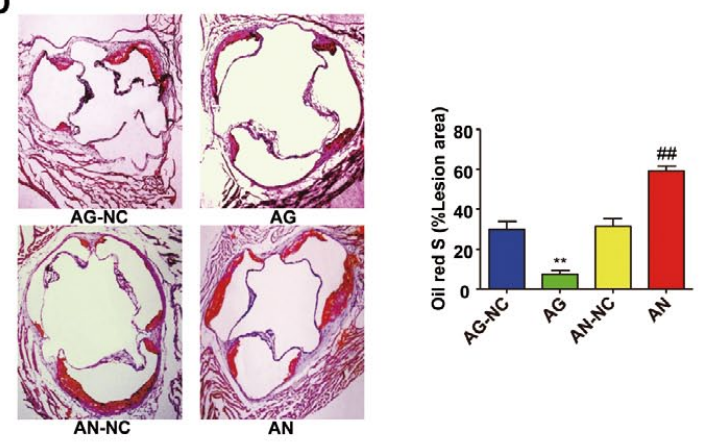

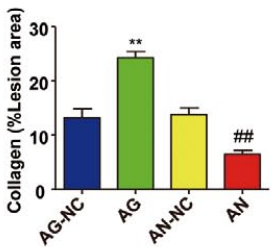

Figure 5. MicroRNA-377 (miR-377) ameliorates the development of atherosclerosis. Atherosclerotic lesions were examined in 12-week-old apolipoprotein $\mathrm{E}$ (ApoE)-knockout (KO) mice that were treated with miR-377 agomir negative control (AG-NC), miR-377 agomir (AG), miR-377 antagomir negative control (AN-NC), or miR-377 antagomir (AN) through tail vein injections. ApoE-KO mice were fed with high fat/high cholesterol diet. (A) Representative images of plaques (blue arrows) in aortic arches and thoracic aortas of ApoE-KO mice. (B) Representative images and quantification of atherosclerotic lesion areas in the en face analysis of the entire aorta with Oil Red O staining. (C) Representative micrographs of hematoxylin-eosin staining in the aortic sinus. (D) Characterization of aortic sinus in atherosclerotic lesion areas by Oil Red O staining. (E) Representative microscopic images and quantification of atherosclerotic plaque collagen content in the aortic sinus by Masson's trichrome staining. Representative images of sections from each group are accompanied by graphs summarizing the data. (Original magnification $\times 10^{4}[\mathbf{C}-\mathbf{E}]$ ). Data are presented as the mean $\pm S E M, n=10$. ${ }^{*} P<0.01$ vs. AG-NC and ${ }^{\# \# P}<0.01$ vs. AN-NC.

Next, we detected the effect of DNMT1 on GPIHBP1 expression. As shown in Figure 3D,E, DNMT1 overexpression increased both activity and protein level of DNMT1. In contrast, DNMT1 inhibition by either 5-Aza-dC or short hairpin DNMT1 (shDNMT1) negatively regulated DNMT1 activity and expression (Figure 3D,E). Most importantly, DNMT1 overexpression inhibited, while DNMT1 inhibition increased GPIHBP1 mRNA and protein levels (Figure 3F,G). Together, these findings indicate that DNMT1 enhances DNA methylation in the promoter region of GPIHBP1, inhibiting GPIHBP1 expression.
miR-377 Decreases GPIHBP1 Promoter Methylation and Increases the Binding of LPL in GPIHBP1-Expressing Cells Considering the inhibitory effect of miR-377 on DNMT1 activity, we examined the effect of miR-377 on GPIHBP1 expression. Treatment with miR-377 mimic significantly decreased DNA methylation of GPIHBP1 and blocked DNMT1-induced increase in DNA methylation of GPIHBP1 (Figure 4A). Conversely, treatment of miR-377 inhibitor increased DNA methylation of GPIHBP1 and restored the inhibitory effects induced by DNMT1 knockdown (Figure 4B). Consistently, miR-377 mimic increased 

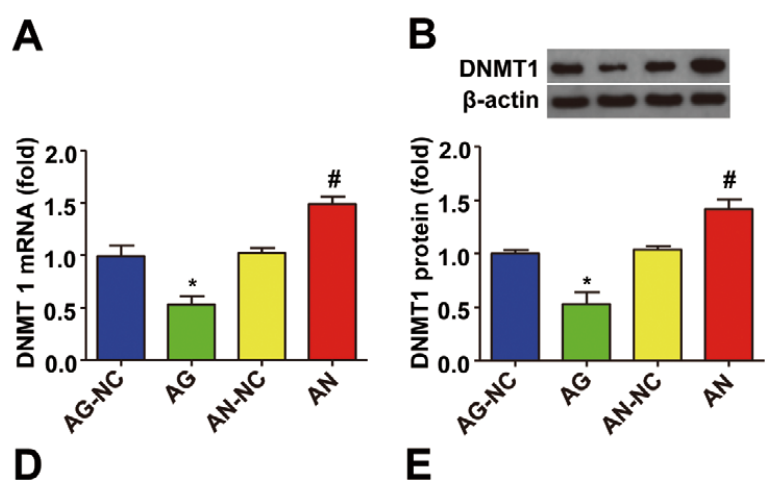

\section{C}

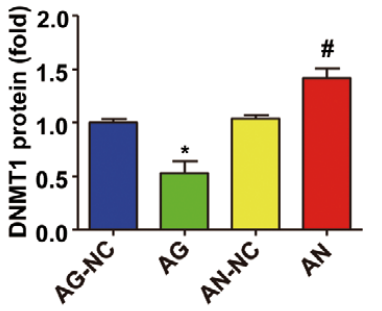

E

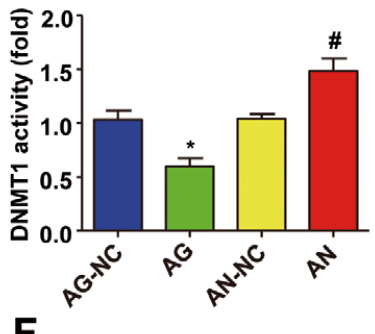

$\mathbf{F}$
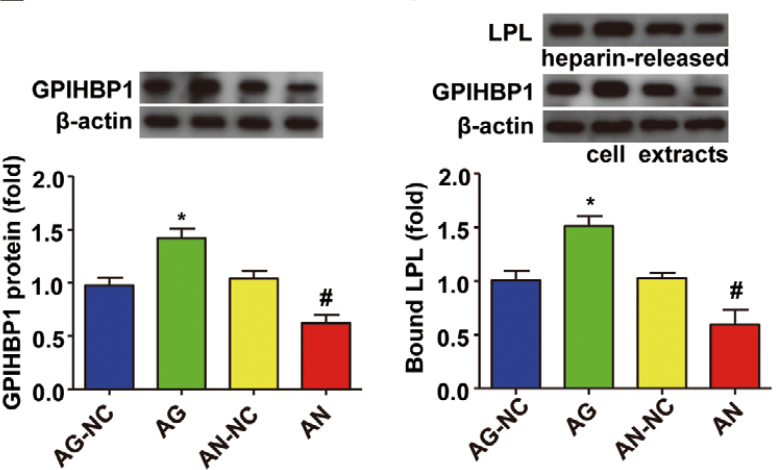

Figure 6. MicroRNA-377 (miR-377) reduces triglyceride levels in apolipoprotein E (ApoE)-knockout (KO) mice. (A-C) DNA methyltransferase 1 (DNMT1) mRNA, protein expression and activity in aortic tissues of ApoE-KO mice treated with miR-377 agomir negative control (AG-NC), miR-377 agomir (AG), miR-377 antagomir negative control (AN-NC), or miR-377 antagomir (AN) via tail vein injections, as determined by quantitative polymerase chain reaction (qPCR) and western blotting analyses respectively. (D,E) Changes in GPIHBP1 mRNA and protein expression in aortic tissues of ApoE-KO mice. (F) The amounts of LPL binding to GPIHBP1 on the surface of mice aorta endothelial cells (MAECs). The heparin-released LPL was examined for the binding of LPL to GPIHBP1 by western blotting (top row). Data are presented as the mean \pm SEM, $n=5$. ${ }^{\star} P<0.05$ vs. AG-NC and \#P<0.05 vs. AN-NC.

(Figure 4C,D), while miR-377 inhibitor reduced the mRNA and protein levels of GPIHBP1 (Figure 4E,F). Overexpression of DNMT1 restored the miR-377-induced reduction in GPIHBP1 methylation (Figure 4A) and increased in the GPIHBP1 expression (Figure 4C,D), while knockdown of DNMT1 essentially eliminated the effects of miR-377 inhibitor on GPIHBP1 methylation and expression (Figure 4B-F). These findings indicate that DNMT1 may mediate the effects of miR-377 on GPIHBP1 expression.

Next, we determined whether miR-377 affected the binding of LPL to GPIHBP1 on the surface of endothelial cells. Endothelial cells were treated with various conditions, as indicated in Figure 4G,H, and then incubated with LPL. Protein expression in cell lysate and heparin-released LPL was detected by Western Blot. miR-377 mimic significantly increased GPIHBP1 expression and the binding of LPL to GPIHBP1 on the cell surface (Figure 4G). Conversely, miR-377 inhibitor decreased GPIHBP1 expression and the levels of bound LPL (Figure 4H). Further, overexpression of DNMT1 reduced, while knockdown of DNMT1 increased, GPIHBP1 expression and the binding of LPL to GPIHBP1 (Figure 4G,H). Importantly, DNMT1 suppressed the miR-377-induced increase in GPIHBP1 expression and the binding of LPL to GPIHBP1 (Figure 4C-G), and knockdown of DNMT1 almost completely suppressed the effect of miR-377 inhibition (Figure 4E-H). These findings suggest the possibility that miR-377 silences DNMT1, leading to increased GPIHBP1 expression and the binding of LPL to GPIHBP1 on the surface of endothelial cell.

\section{miR-377 Inhibits the Development of Atherosclerosis in ApoE-KO Mice}

We further examined the effects of miR-377 on the development of atherosclerosis in ApoE-KO mice. Mice treated with $\mathrm{AG}$ displayed a significant reduction in atherosclerotic plaque area, while AN treatment increased plaque areas when compared with their negative controls (Figure 5A). We also observed that the severity of lipid deposition in the atherosclerotic plaque of aortas of mice was significantly decreased in AG-treated mice, but increased in AN-treated mice (Figure 5B). We further investigated the effects of miR-377 on plaque formation, lipid deposition, and collagen content in aortic sinus. Similarly, the aortic sinus plaque area and lipid deposition were much less than in AG-treated mice, but much greater in AG-NC-treated mice when compared to their control groups (Figure 5C,D). Masson's trichrome staining results showed that the collagen content in aortic sinus was increased in AG-treated mice but reduced in AN-treated mice, suggesting that miR-377 increases the stability of atherosclerotic plaque at the aortic sinus (Figure 5E). Together, the results indicated the anti-atherogenic role of miR-377 in ApoE-KO mice. 


\begin{tabular}{|lccccc|}
\hline \multicolumn{5}{l}{ Table. Effects of miR-377 on Plasma Lipid Levels in Apolipoprotein E-Knockout Mice } \\
AG-NC & BW (g) & TG & TC & HDL-C & LDL-C \\
AG & $28.21 \pm 0.97$ & $2.56 \pm 0.25$ & $19.38 \pm 0.75$ & $3.21 \pm 0.58$ & $15.96 \pm 0.53$ \\
AN-NC & $27.48 \pm 1.26$ & $1.24 \pm 0.38^{*}$ & $16.37 \pm 1.06^{*}$ & $3.13 \pm 0.63$ & $12.52 \pm 1.50^{*}$ \\
AN & $28.57 \pm 1.33$ & $2.47 \pm 0.29$ & $18.86 \pm 0.82$ & $3.02 \pm 0.65$ & $16.02 \pm 0.74$ \\
\end{tabular}

Data are presented as the mean \pm SEM, $n=10 .{ }^{*} \mathrm{P}<0.05$ vs. agomir negative control (AG-NC) and ${ }^{\#} \mathrm{P}<0.05$ vs. antagomir negative control (AN-NC). Plasma from the different experimental groups were measured enzymatically. BW, body weight; HDL-C, high-density lipoprotein cholesterol; LDL-C, low-density lipoprotein cholesterol; miR-377, microRNA-377; TC, total cholesterol; TG, triglyceride.

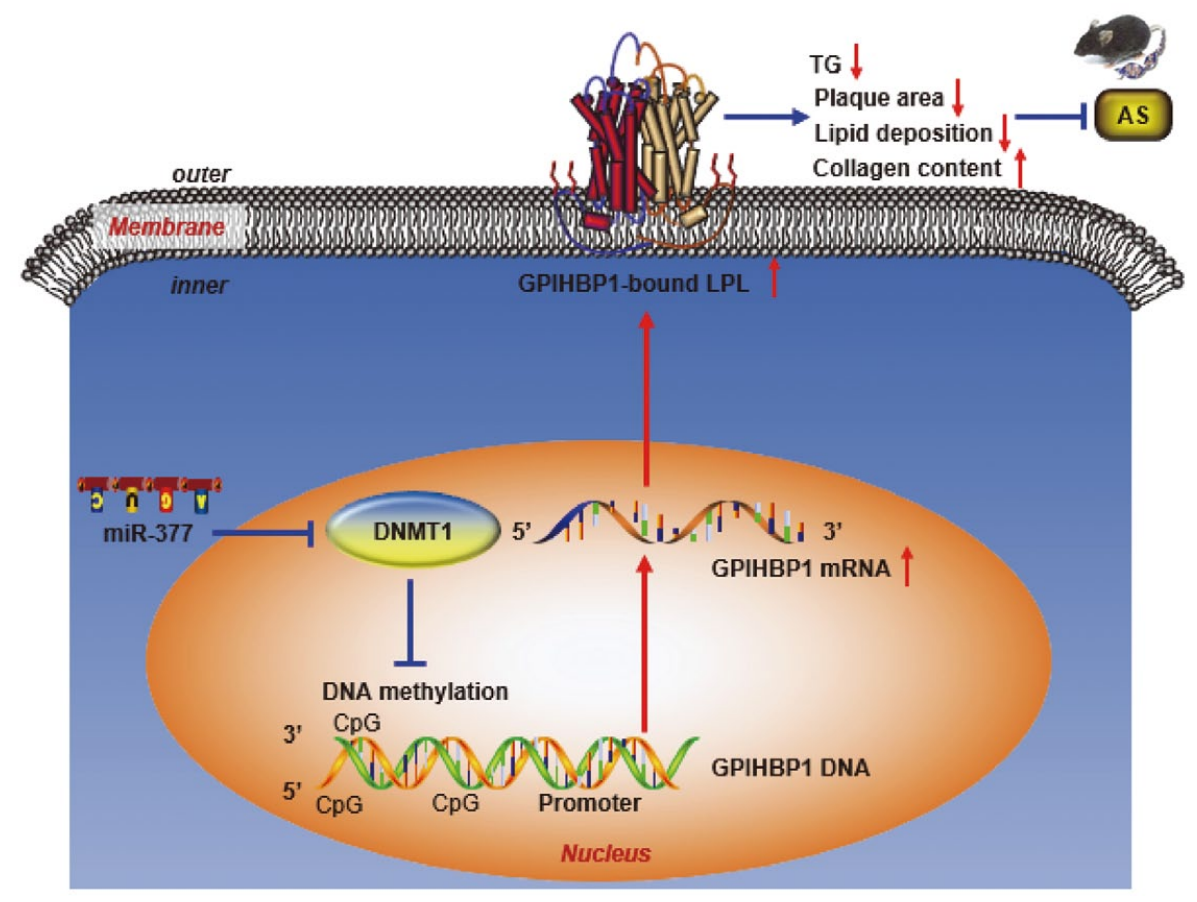

Figure 7. Model of the mechanism underlying microRNA-377 (miR-377) regulation of triglyceride levels and prevention of atherosclerosis in apolipoprotein E (ApoE)-knockout (KO) mice. DNA methyltransferase 1 (DNMT1) catalyzes glycosylphosphatidylinositol-anchored high-density lipoprotein-binding protein 1 (GPIHBP1) promoter DNA methylation, and miR-377 inhibits DNMT1 activity and protein expression. Consequently, miR-377 treatment enhances the binding of lipoprotein lipase (LPL) to GPIHBP1 in the endothelial cell surface by repressing DNMT1. Endothelial LPL plays an important role in hydrolyzing triglycerides (TG) in plasma. Taken together, the miR-377-DNMT1-GPIHBP1 cascade plays a vital role in the regulation of triglyceride levels and prevention of atherosclerosis (AS).

\section{miR-377 Reduces Triglyceride Levels Through the DNMT1-GPIHBP1-LPL Axis in ApoE-KO Mice}

To investigate the potential contribution of the DNMT1GPIHBP1-LPL pathway to the effect of miR-377 on the development of atherosclerosis in ApoE-KO mice, we determined the expression of DNMT1 and GPIHBP1 in the aortic arch of mice in each group. AG treatment markedly decreased, while AN treatment increased the levels of DNMT1 mRNA, protein expression and activity in mice (Figure 6A-C). As expected, the levels of GPIHBP1 mRNA and protein expression, and the binding of LPL to GPIHBP1 on the surface of aorta endothelial cells (MAECs) were increased in AG-treated mice, but decreased in AN-treated mice (Figure 6D-F). AG treatment also significantly reduced plasma levels of TG, TC, and LDL-C, but had no effect on plasma HDL-C in ApoE-KO mice fed the Western-type diet. AN treatment also did not change plasma levels of HDL-C, but significantly increased plasma levels of TG, TC, and LDL-C (Table). These data indicate that miR-377 might regulate plasma triglyceride levels via the miR377-DNMT1-GPIHBP1-LPL axis in ApoE-KO mice.

\section{Discussion}

In this study, we have demonstrated the roles of miR-377 and DNMT1 in triglyceride metabolism and in the development of atherosclerosis. Our findings revealed that miR-377 reduced DNA methylation in GPIHBP1 promoter through directly targeting DNMT1, thereby increasing GPIHBP1 expression and the binding of LPL to GPIHBP1 
on the surface of endothelial cells. Besides, miR-377 reduced plasma triglyceride levels and suppresses the development of atherosclerosis in ApoE-KO mice via the DNMT1/ GPIHBP1/LPL pathway. These findings may have implications for the therapeutic prevention of hypertriglyceridemia and atherosclerosis.

To the best of our knowledge, this is the first report on epigenetic regulation of GPIHBP1 by DNMT1. The GPIHBP1 promoter region contains two $\mathrm{CpG}$ islands that are characterized by a length greater than $200 \mathrm{bp}, \mathrm{GC}$ content greater than $50 \%$ and $\mathrm{ObsCpG/ExpCpG}>0.60$. Our data showed that DNMT1 effectively catalyzed GPIHBP1 promoter DNA methylation, which was inhibited by a DNMT1 inhibitor, 5-Ara-dC, or knockdown of DNMT1 expression. GPIHBP1, as a GPI-anchored protein of capillary endothelial cells, binds LPL stably in the sub-endothelial space and then transports LPL to the capillary lumen..$^{21}$ LPL promotes hydrolysis of TG on TRLs migrated to the surface of capillary cells..$^{29}$ In GPIHBP1deficient mice, LPL binds to heparan sulfate proteoglycans (HSPG) and accumulates in the interstitial space. ${ }^{30}$ Basically, GPIHBP1 offers a binding site for LPL in the capillary lumen and creates "a platform for lipolysis". We showed that DNMT1 downregulated GPIHBP1 expression through enhancing DNA methylation in the GPIHBP1 promoter region, suggesting that aberrant methylation of GPIHBP1 promoter may associate with hypertriglyceridemia.

Recently, 2 publications showed that miR-377 modulated senescence in human skin fibroblasts and reversed cancerous phenotypes of pancreatic cells through inhibiting DNMT1. ${ }^{31,32}$ Consistently, our study revealed that miR-377 contained 8 bases that were predicted to complementary pair with DNMT1 UTR. Consistently, miR-377 directly targeted DNMT1 3'-UTR and suppressed its expression and activity. Despite the fact that epigenetic modifications such as microRNAs regulation have been shown to regulate DNMT1, the regulatory role of miR-377 in DNMT1 expression and activity in triglyceride metabolism is unclear. Except for DNMT1, DNMTs also include DNMT2 and DNMT3. ${ }^{15}$ Recently, increasing studies has shown that either DNMT2 or DNMT3 plays an important role in global DNA methylation. ${ }^{33-35}$ Accordingly, DNMT2 and DNMT3 may also have effects on GPIHBP1 promoter DNA methylation. Further work will be required to dissect whether DNMT2 or DNMT3 affects DNA methylation of the GPIHBP1 promoter.

A recent study demonstrated that miR-377 can be used as early biomarkers of nephropathy and subclinical atherosclerotic risk in pediatric patients with type I diabetes. ${ }^{12}$ Here, we found that plasma miR-377 mRNA expression was significantly decreased in adult patients with hypertriglyceridemia. It was reported that miR-377 expression was repressed by a high-fat diet, indicating that miR-377 may regulate triglyceride metabolism and affect the development of atherosclerosis.11 MiR-145, miR-410 and miR-377 mRNA levels are all decreased in patients with hyperlipidemia. Detailed analysis revealed that only miR-377 mRNA expression was significantly reduced in patients with high triglyceride levels. Recently, miR-145 was reported to be associated with hyperglycemia in diabetes. ${ }^{36}$ Thus, reduced plasma miR-145 levels observed in our patient samples may be related to increased fasting blood glucose levels. In contrast, miR-410 may be more closely related to plasma cholesterol level. Next, we investigated whether miR-377 increased the binding of LPL to GPIHBP1 through the downregulation of DNMT1. The LPL and GPIHBP1 binding assay showed that miR-377 mimic increased, while miR-377 inhibitor markedly decreased the binding of LPL to GPIHBP1, which were blocked by overexpression or knockdown of DNMT1. This suggests that miR-377 mediates triglyceride metabolism by inhibiting DNMT1. Moreover, miR-377 reduces plasma triglyceride levels and significantly decreases the area of aortic atherosclerotic lesion and necrotic core in ApoE-KO mice. These findings suggest that miR-377 is an important contributor to the inhibition of hypertriglyceridemia and the development of atherosclerosis.

Collectively, our results strongly demonstrate that miR-377 directly inhibits DNMT1 and subsequently downregulates DNA methylation levels of the GPIHBP1 promoter, increasing GPIHBP1 expression and the binding of LPL to GPIHBP1. This reduces plasma triglyceride levels and suppresses the development of atherosclerosis (Figure 7). These data have shed new light on the mechanism underlying epigenetic regulation of GPIHBP1, and may provide a new target in regulating triglyceride metabolism and preventing atherosclerosis.

\section{Acknowledgments}

We gratefully acknowledge the patients for their participation in this study. This work was supported by grants from the National Natural Sciences Foundation of China (81570408), University of South China Innovation Foundation for Postgraduate (2017XCX03) and the construct program of the key discipline in Hunan Province, China (Basic Medicine Sciences in University of South China, Xiangjiaofa, No. [2011]76).

\section{Conflicts of interest}

The authors declare no actual or potential conflicts of interest.

\section{References}

1. Ariza MJ, Martinez-Hernandez PL, Ibarretxe D, Rabacchi C, Rioja J, Grande-Araqón C, et al. Novel mutations in the GPIHBP1 gene identified in 2 patients with recurrent acute pancreatitis. J Clin Lipidol 2016; 10: $92-100$, e101.

2. Ding Y, Zhang L, Wang Y, Huang W, Tang Y, Bai L, et al. Amelioration of hypertriglyceridemia with hypo-alpha-cholesterolemia in LPL deficient mice by hematopoietic cell-derived LPL. PLoS One 2011; 6: e25620.

3. Wojciechowska A, Braniewska A, Kozar-Kaminska K. MicroRNA in cardiovascular biology and disease. Adv Clin Exp Med 2017; 26: $865-874$.

4. Ceolotto G, Giannella A, Albiero M, Kuppusamy M, Radu C, Simioni $\mathrm{P}$, et al. miR-30c-5p regulates macrophage-mediated inflammation and pro- atherosclerosis pathways. Cardiovasc Res 2017; 113: $1627-1638$.

5. Tian GP, Tang YY, He PP, Lv YC, Ouyang XP, Zhao GJ, et al. The effects of miR-467b on lipoprotein lipase (LPL) expression, pro-inflammatory cytokine, lipid levels and atherosclerotic lesions in apolipoprotein E knockout mice. Biochem Biophys Res Commun 2014; 443: 428-434.

6. Lan G, Xie W, Li L, Zhang M, Liu D, Tan YL, et al. MicroRNA-134 actives lipoprotein lipase-mediated lipid accumulation and inflammatory response by targeting angiopoietin-like 4 in THP-1 macrophages. Biochem Biophys Res Commun 2016; 472: $410-417$.

7. Yao Y, Zhang X, Chen HP, Li L, Xie W, Lan G, et al. MicroRNA-186 promotes macrophage lipid accumulation and secretion of pro-inflammatory cytokines by targeting cystathionine gamma-lyase in THP-1 macrophages. Atherosclerosis 2016; 250: $122-132$.

8. Joladarashi D, Garikipati VN, Thandavarayan RA, Verma SK, Mackie AR, Khan M, et al. Enhanced cardiac regenerative ability of stem cells after ischemia- reperfusion injury: Role of human CD34+ cells deficient in microRNA-377. $J$ Am Coll Cardiol 2015; 66: 2214-2226.

9. Wang W, Ding XQ, Gu TT, Song L, Li JM, Xue QC, et al. 
Pterostilbene and allopurinol reduce fructose-induced podocyte oxidative stress and inflammation via microRNA-377. Free Radic Biol Med 2015; 83: 214-226.

10. Wen Z, Huang W, Feng Y, Cai W, Wang Y, Wang X, et al. MicroRNA-377 regulates mesenchymal stem cell-induced angiogenesis in ischemic hearts by targeting VEGF. PLoS One 2014; 9: e104666.

11. Casas-Agustench P, Fernandes FS, Tavares do Carmo MG, Visioli F, Herrera E, Dávalos A. Consumption of distinct dietary lipids during early pregnancy differentially modulates the expression of microRNAs in mothers and offspring. PLoS One 2015; 10: 0117858 .

12. El-Samahy MH, Adly AA, Elhenawy YI, Ismail EA, Pessar SA, Mowafy ME, et al. Urinary miRNA-377 and miRNA-216a as biomarkers of nephropathy and subclinical atherosclerotic risk in pediatric patients with type 1 diabetes. J Diabetes Complications 2018; 32: 185-192.

13. Hernando-Herraez I, Heyn H, Fernandez-Callejo M, Vidal E, Femandez-Bellon H, Prodo-Martinez J, et al. The interplay between DNA methylation and sequence divergence in recent human evolution. Nucleic Acids Res 2015; 43: 8204-8214.

14. Hernando-Herraez I, Garcia-Perez R, Sharp AJ, Marques-Bonet T. DNA methylation: Insights into human evolution. PLoS Genet 2015; 11: e1005661.

15. Lan J, Hua S, He X, Zhang Y. DNA methyltransferases and methyl-binding proteins of mammals. Acta Biochim Biophys Sin (Shanghai) 2010; 42: 243-252.

16. Yu J, Qiu Y, Yang J, Bian S, Chen G, Deng M, et al. DNMT1PPARgamma pathway in macrophages regulates chronic inflammation and atherosclerosis development in mice. Sci Rep 2016; 6: 30053 .

17. Gao J, Qiu X, Wang X, Peng C, Zheng F. Associations of ChREBP and global DNA methylation with genetic and environmental factors in Chinese healthy adults. PLoS One 2016; 11: e0157128

18. He B, Yin C, Gong Y, Liu J, Guo H, Zhao R. Melatonininduced increase of lipid droplets accumulation and in vitro maturation in porcine oocytes is mediated by mitochondrial quiescence. J Cell Physiol 2017; 233: 302-312.

19. Chi X, Shetty SK, Shows HW, Hjelmass AJ, Malcolm EK, Davies BS. Angiopoietin-like 4 modifies the interactions between lipoprotein lipase and its endothelial cell transporter GPIHBP1. J Biol Chem 2015; 290: 11865-11877.

20. Young SG, Davies BS, Fong LG, Gin P, Weinstein MM, Bensadoun A, et al. GPIHBP1: An endothelial cell molecule important for the lipolytic processing of chylomicrons. Curr Opin Lipidol 2007; 18: 389-396.

21. Davies BS, Beigneux AP, Barnes RH 2nd, Tu Y, Gin P, Weinstein MM, et al. GPIHBP1 is responsible for the entry of lipoprotein lipase into capillaries. Cell Metab 2010; 12: 42-52.

22. Beigneux AP, Davies BS, Gin P, Weinstein MM, Farber E, Qiao $\mathrm{X}$, et al. Glycosylphosphatidylinositol-anchored high-density lipoprotein-binding protein 1 plays a critical role in the lipolytic processing of chylomicrons. Cell Metab 2007; 5: 279-291.

23. Li Y, He PP, Zhang DW, Zheng XL, Cayabyab FS, Yin WD, et al. Lipoprotein lipase: From gene to atherosclerosis. Atherosclerosis 2014; 237: 597-608.

24. Sonnenburg WK, Yu D, Lee EC, Xiong W, Gololobov G, Key $\mathrm{B}$, et al. GPIHBP1 stabilizes lipoprotein lipase and prevents its inhibition by angiopoietin-like 3 and angiopoietin-like 4 . J Lipid Res 2009; 50: $2421-2429$.

25. Guay SP, Gaudet D, Brisson D. The g. $-469 \mathrm{G}>$ A polymorphism in the GPIHBP1 gene promoter is associated with hypertriglyceridemia and has an additive effect on the risk conferred by LPL defective alleles. Nutr Metab Cardiovasc Dis 2013; 23: 358-365.
26. Buonuomo PS, Bartuli A, Rabacchi C, Bertolini S, Calandra S A 3-day-old neonate with severe hypertriglyceridemia from novel mutations of the GPIHBP1 gene. J Clin Lipidol 2015; 9: 265-270.

27. Beigneux AP, Miyashita K, Ploug M, Blom DJ, Ai M, Linton $\mathrm{MF}$, et al. Autoantibodies against GPIHBP1 as a cause of hypertriglyceridemia. $N$ Engl J Med 2017; 376: 1647-1658.

28. Cheng HP, Gong D, Zhao ZW, He PP, Yu XH, Ye Q, et al. MicroRNA-182 promotes lipoprotein lipase expression and atherogenesis by targeting histone deacetylase 9 in apolipoprotein E-knockout mice. Circ J 2017; 82: 28 - 38.

29. Goulbourne CN, Gin P, Tatar A, Nobumori C, Hoenger A, Jiang H, et al. The GPIHBP1-LPL complex is responsible for the margination of triglyceride-rich lipoproteins in capillaries. Cell Metab 2014; 19: 849-860.

30. Mysling S, Kristensen KK, Larsson M, Beiqneux AP, Gårdsvoll H, Fong LG, et al. The acidic domain of the endothelial membrane protein GPIHBP1 stabilizes lipoprotein lipase activity by preventing unfolding of its catalytic domain. Elife 2016; 5: e12095.

31. Xie HF, Liu YZ, Du R, Wang B, Chen MT, Zhang YY, et al. miR-377 induces senescence in human skin fibroblasts by targeting DNA methyltransferase 1. Cell Death Dis 2017; 8: e2663.

32. Azizi M, Fard-Esfahani P, Mahmoodzadeh H, Fazeli MS, Azadmanesh K, Zeinali S, et al. MiR-377 reverses cancerous phenotypes of pancreatic cells via suppressing DNMT1 and demethylating tumor suppressor genes. Epigenomics 2017; 9: $1059-1075$

33. Ferreira HJ, Heyn H, Vizoso M, Moutinho C, Vidal E, Gomez A, et al. DNMT3A mutations mediate the epigenetic reactivation of the leukemogenic factor MEIS1 in acute myeloid leukemia. Oncogene 2016; 35: 3079-3082.

34. $\mathrm{Lu} \mathrm{W}, \mathrm{Lu} \mathrm{T}$, Wei X. Downregulation of DNMT3a expression increases miR-182- induced apoptosis of ovarian cancer through caspase-3 and caspase-9-mediated apoptosis and DNA damage response. Oncol Rep 2016; 36: 3597-3604.

35. Zhang Y, Sun X, Zhang L, Zhang W. Testicular Dnmt3 expression and global DNA methylation are down-regulated by gonadotropin releasing hormones in the ricefield eel Monopterus albus. Sci Rep 2017; 7: 43158 .

36. Wang BW, Fang WJ, Shyu KG. MicroRNA-145 regulates disabled-2 and Wnt3a expression in cardiomyocytes under hyperglycaemia. Eur J Clin Invest 2018; 48: e12867.

\section{Supplementary Files}

\section{Supplementary File 1}

\section{Supplementary Methods}

Figure S1. MicroRNA-377 (miR-377) directly targets the 3'-untranslated region (3'-UTR) of DNA methyltransferase 1 (DNMT1).

Figure S2. Bioinformatic analyses of the potential methylation sites of glycosylphosphatidylinositol-anchored high-density lipoprotein binding protein 1 (GPIHBP1) promoter.

Table S1. Primer sequence for real-time polymerase chain reaction analysis

Table S2. Characteristics of patients with or without hyperlipidemia among coronary artery disease

Table S3. Comparison of the human GPIHBP1 CGI with standard CGI definitions

Please find supplementary file(s);

http://dx.doi.org/10.1253/circj.CJ-18-0410 